\title{
Suppository Dissolution Utilizing USP Apparatus 4
}

Standardized in-vitro dissolution testing of suppositories has been of interest to the pharmaceutical industry since the early works of Giabaldi and Gundhofer in 1975.(1)

Anthony Palmieri examined standardizing suppository dissolutions in his work at the University of Wyoming (2). Palmieri developed a slotted dissolution basket for suppositories that is

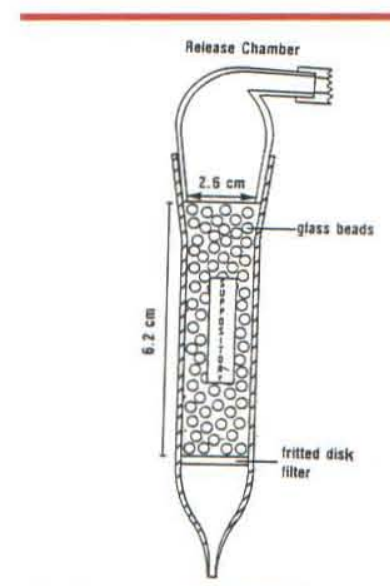

Figure 1. Schematic of Roseman's continuous flow bead-bed dissolution apparatus (from reference 3 ) widely used by many individuals in the pharmaceutical industry. The slotted basket, however, is not suitable for all suppository bases. Palmieri's basket does not perform suitably in those cases where the formulation contained a base with a relatively low melting point. The problem that exists is the lack of control over the surface area of the suppository as it begins to melt. This is of concern because the amount of interfacial area exposed to the dissolution media directly effects the release rate of the drug.(3) It is necessary to evaluate alternative dissolution methodologies in these particular cases.

In this study, commercial Acetaminophen suppositories were used to evaluate the concept of transferring the methodology of con-

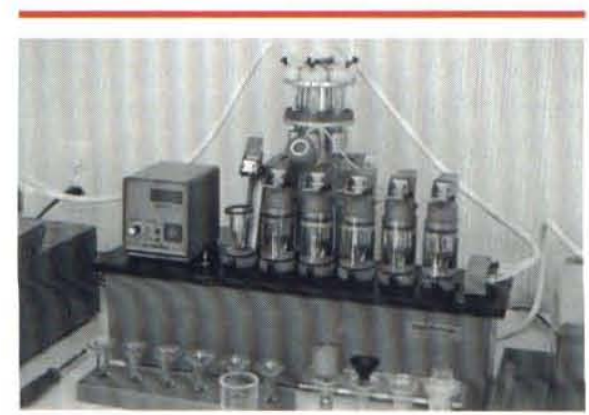

Figure 2. USP Dissolution Apparatus 4. tinuous flow bead-bed dissolution to USP Dissolution Apparatus 4. Continuous flow bead-bed dissolution methodology was extensively evaluated and shown to be a value in suppository dissolutions by Roseman, et al.(3) In his work, Roseman used glass beads to suspend a suppository in a cell while media

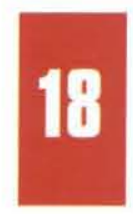
passed over the suppository (Figure 1). The glass beads in this system control the interfacial area of the suppository. USP Dissolution Apparatus 4 contains the basic design and principles of Roseman's original apparatus.

\section{Ronald Dunn, Hilda Reimers, Lisa Ward and James Chapman \\ Upsher-Smith, Minneapolis, $M N$}

In our study, a Sotax CE6, Dissotest ${ }^{\oplus}$ apparatus was used (Figure 2 ). A closed system was designed by placing $900 \mathrm{~mL}$ of USP Purified Water in a $2 \mathrm{~L}$ flask. The flask was maintained at a temperature of $37.0^{\circ} \mathrm{C} \pm 0.5^{\circ} \mathrm{C}$ in a water bath. The dissolution media (USP Purified Water) was drawn from the flask, through the cell and returned to the flask. The

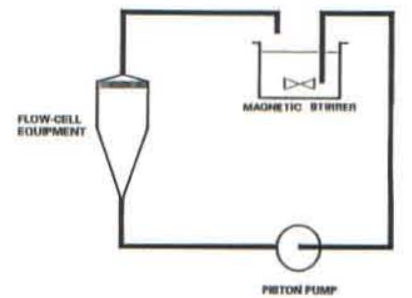

Figure 3. Dissotest Closed System media was continuously stirred throughout the dissolution using a stir bar (Figure 3). The $22.0 \mathrm{~mm}$ Dissotest cell was prepared by placing a $5 \mathrm{~mm}$ ruby bead in the bottom of the cell and filling one-third of the way with $1 \mathrm{~mm}$ glass beads. Glass beads with a diameter of $4 \mathrm{~mm}$ were used to fill the

Figure 4. $22.0 \mathrm{~mm}$ Flow Cell

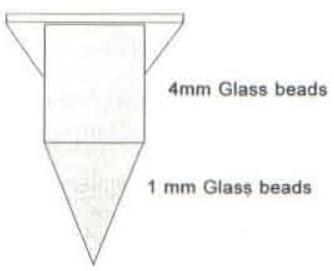

remainder of the cell volume. The suppository was then placed tip down in the center of the cell until the tip just reached the $1 \mathrm{~mm}$ glass beads (Figure 4). The cell was placed in the Dissotest ${ }^{\infty}$ apparatus and the pump started. The flow rate was controlled at $20 \pm 0.5 \mathrm{~mL} / \mathrm{min}$.

Drug release was monitored by collecting $20 \mathrm{~mL}$ samples using a Luer lock syringe fitted with a dissolution sampling device and filter. Media was replaced after each collection. The collected samples were analyzed using HPLC. The amount of acetaminophen released was calculated and the dissolution rate graphed.

Figure 5 illustrates the dissolution profiles (average of $n=3$ ) of acetaminophen suppositories run on three separate days. The average relative standard deviation over all time points for each 


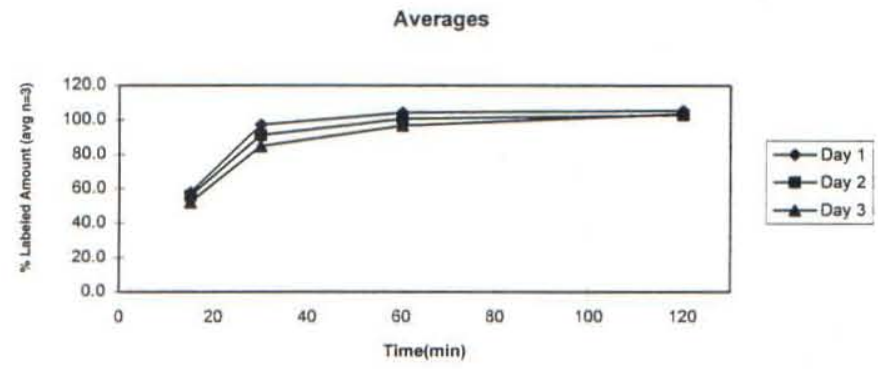

Figure 5. Dissolution rates of acetaminophen suppositories using USP Apparatus 4.

individual suppository was $6.73 \%$. The dissolution results yielded higher relative standard deviations in the initial time points but became more reproducible in the later stages (Table 1).

\begin{tabular}{|c|c|c|}
\hline Time (min) & Standard Deviation & \%RSD \\
\hline \hline 15 & 7.275 & 13.151 \\
\hline 30 & 6.524 & 7.159 \\
\hline 60 & 4.055 & 4.031 \\
\hline 120 & 2.683 & 2.577 \\
\hline
\end{tabular}

Table 1

The data indicates that this apparatus may be suitable for quality control monitoring of finished product. Further studies are being conducted to evaluate this system for quality control monitoring, stability indicating abilities, and in-vivo correlation.

\section{References}

1.Gibaldi, M., Gundhofer, J., 7. Pharm. Sci., 64:1064 (June 1975)

2.Palmieri, A., Drug Development and Industrial Pharmacy, 7:2,247-259 (1981)

3.Roseman, et. al., f. Pbarm. Sci., 70:646 (1981) 